\title{
Avaliando a comutatividade: importante requisito da qualidade para laboratórios clínicos
}

Primeira submissão em 25/02/11 Última submissão em 27/09/11 Aceito para publicação em 28/09/11 Publicado em 20/12/11

\section{Understanding commutability: important quality requirement for clinical laboratories}

Chams Bicalho Maluf'; Indiayara Oliveira Silva²; Pedro Guatimosim Vidigal ${ }^{3}$

\section{unitermos \\ Contagem de células \\ sanguíneas}

Técnicas e procedimentos de laboratório

Controle da qualidade

Acreditação

Gestão em saúde

Comutatividade

\section{resumo}

Introdução: Poucos laboratórios no Brasil realizam a avaliação da comutatividade dos resultados de exames, provavelmente por desconhecimento dos procedimentos para sua realização e também pela pouca importância dada a essa avaliação. Objetivo: Avaliar a comutatividade dos resultados de exames hematológicos realizados em três analisadores automatizados em um laboratório de um hospital público universitário, em Belo Horizonte, MG, Brasil, propondo procedimento prático, de baixa complexidade, factível de ser utilizado em laboratórios clínicos. Material e métodos: As amostras foram selecionadas visando obter valores nos níveis de decisão terapêutica. Foram processadas seis amostras por dia, durante quatro dias, em duplicata em três analisadores, totalizando 48 replicatas em cada instrumento. Foi avaliada a correlação entre os resultados de 10 dos principais parâmetros hematológicos obtidos nos instrumentos-teste e aqueles obtidos com o instrumento-referência. Foram estimados os erros sistemáticos e totais, considerando as especificações da variação biológica como limite máximo aceitável. Resultados: O coeficiente de correlação $(r)$ entre os equipamentos para os parâmetros investigados foi $>0,975$. Os erros sistemático (médio) e total, obtidos para os parâmetros analisados, quando se comparam os equipamentos de teste com o de referência, atenderam às especificações da qualidade definidos. Discussão: A comutatividade é um importante processo da gestão da qualidade do laboratório clínico e visa garantir a comparabilidade de resultados de exames realizados por diferentes sistemas. Conclusão: Utilizando procedimento padronizado internacionalmente, prático e de baixa complexidade, demonstrou-se que os exames realizados nos equipamentos avaliados são equivalentes, podendo ser usados indistintamente no acompanhamento de pacientes.

\section{abstract}

Introduction: Few Brazilian laboratories evaluate the commutability of test results, probably due to the lack of procedure expertise as well as the neglected importance of its assessment. Objective: The aim of this study was to evaluate the commutability of hematological test results performed with three automated analyzers at the laboratory of a public university hospital in Belo Horizonte, MG, Brazil, proposing a practical, simple, and feasible procedure to be applied in clinical laboratories. Material and methods: Samples were selected in order to obtain hematologic values for therapeutic decision levels. Six samples were processed in duplicate in three analyzers daily during a four-day period amounting to a total of 48 replicates in each instrument. The correlation between the results of 10 hematologic parameters obtained with test instruments and the reference instrument was assessed. Systematic and total errors were estimated and criteria for acceptable performance were based on the biological variation specifications. Results: The correlation coefficient ( $r$ ) between test instruments and reference instrument results was $>0.975$. Systematic (mean) and total errors met the required quality specifications when compared with reference instruments. Discussion: Commutability is an important process of quality management in clinical laboratories and it ensures the comparability of test results carried out with different procedures. Conclusion: Through a practical, simple, and internationally standardized procedure, this study showed that test results from the evaluated instruments were equivalent, which allows their use in patient monitoring. key words

Blood cell count

Laboratory techniques and procedures

Quality control

Accreditation

Health management

Commutability

1. Médica; mestranda do Programa de Pós-Graduação em Patologia da Faculdade de Medicina da Universidade Federal de Minas Cerais (UFMG).

2. Biomédica; bolsista de iniciação científica do Laboratorium - Núcleo de Pesquisa, Educação e Assessoria em Cestão Laboratorial da Faculdade de Medicina da UFMG.

3. Doutor em Medicina; professor associado de Patologia Clínica da UFMG ; coordenador do Laboratorium - Núcleo de Pesquisa, Educação e Assessoria em Cestão Laboratorial da Faculdade de Medicina da UFMG. 


\section{Introdução}

O termo controle da qualidade, introduzido nos laboratórios clínicos décadas atrás, refere-se ao "controle estatístico da qualidade que é comumente usado nos laboratórios para monitorar o desempenho dos testes processados na rotina, detectando possíveis erros, e corrigindo problemas antes dos resultados serem liberados" ${ }^{\prime \prime 18)}$. Os termos controle interno e controle externo da qualidade referem-se aos programas usados para avaliar, de forma contínua, a qualidade analítica ${ }^{(16)}$.

Assim, com a finalidade de garantir a efetividade dos processos analíticos e atendendo às expectativas e necessidades dos clientes e conformidades legais, os laboratórios devem manter rotinas do controle interno da qualidade, que permitem a verificação da variabilidade das medições (precisão), e participar de programas externos de controle da qualidade, que permitem monitorar a capacidade do método de apresentar resultados próximos do valor verdadeiro (exatidão) $^{(8)}$. Já os programas de acreditação de laboratórios clínicos têm entre seus principais objetivos, além de monitorar a qualidade, garantir a melhoria contínua dos serviços prestados pelos laboratórios clínicos.

Outra importante ação de controle da qualidade do laboratório clínico é a avaliação periódica da comutatividade, que visa monitorar a equivalência de resultados de exames quando realizados em diferentes equipamentos ou sistemas analíticos. A análise da comutatividade também deve ser incluída na validação de novos métodos, sistemas ou equipamentos, precedendo a implementação de seu uso na rotina. Dessa forma, é possível verificar se o novo método, sistema ou equipamento atende às especificações da qualidade e se seus resultados são equivalentes ao método, sistema ou equipamento já em uso no laboratório clínico.

O termo comutabilidade foi inicialmente usado para definir a capacidade de possuir propriedades interensaios comparáveis em materiais de referência, de calibração ou de controle, demonstradas por amostras clínicas autênticas, quando medidas por mais de um sistema analítico ${ }^{(14,15)}$. Documentos da International Standardization Organization (ISO) expandiram esse conceito, descrevendo comutabilidade como a equivalência das relações matemáticas entre os resultados de diferentes sistemas analíticos de medida para um material de referência e para amostras representativas de indivíduos saudáveis ou doentes para determinado analito ${ }^{(3,}$ 5). No glossário da versão 2007 da norma do Programa de Acreditação de Laboratórios Clínicos (PALC) da Sociedade Brasileira de Patologia Clínica/Medicina Laboratorial (SBPC) $\mathrm{ML})$, comutatividade foi definida como a capacidade de- monstrável estatisticamente ou de outra forma de que dois ou mais sistemas analíticos geram, para as mesmas amostras de pacientes, resultados clinicamente equivalentes ${ }^{(11)}$. Na versão 2010 dessa norma, esse conceito passou a ser atribuído ao termo equivalência ${ }^{(12)}$. Assim, as nomenclaturas comutatividade, comutabilidade (commutability) e equivalência de sistemas analíticos parecem não estar normalizadas, podendo ser usadas de forma indistinta.

A exigência da avaliação da comutatividade nos requisitos de programas de acreditação, como o PALC, destaca sua importância, muitas vezes negligenciada pelos laboratórios ${ }^{(11,12)}$. Não existem dados disponíveis que permitam determinar o número de laboratórios que incluem a avaliação da comutatividade na gestão da qualidade. Considerando os 97 laboratórios acreditados pela norma PALC e o número de laboratórios clínicos atuantes no Brasil (aproximadamente $17 \mathrm{mil}$ ), mesmo excluindo aqueles que não realizam exames por mais de um método, sistema ou equipamento, parece que a avaliação da comutatividade é pouco realizada ${ }^{(2,13)}$. Possíveis explicações para a não realização dessa avaliação incluem o desconhecimento de sua importância e a pouca familiaridade com as ferramentas e os procedimentos necessários para sua realização. Além disso, apesar do grande número de publicações que abordam o controle da qualidade no laboratório clínico, são escassas aquelas que estudam o tema da comutabilidade ou equivalência de resultados laboratoriais ${ }^{(9)}$.

Este estudo visou avaliar a comutatividade dos resultados de exames hematológicos realizados em três analisadores automatizados em um laboratório de um hospital público universitário, em Belo Horizonte, Minas Gerais, Brasil, propondo um procedimento internacionalmente padronizado, de baixa complexidade, factível de ser utilizado em laboratórios clínicos.

\section{Material e métodos}

Foram utilizados três analisadores hematológicos automatizados disponíveis no laboratório, marca Sysmex, modelo XE-2100D (Kobe, Japão), disponíveis no Laboratório do Hospital das Clínicas da Universidade Federal de Minas Gerais (UFMG). No presente estudo, os instrumentos foram denominados: analisador hematológico A (AHA), definido como instrumento-referência, com o qual os demais (analisador hematológico $B[A H B]$ e analisador hematológico $C$ $[\mathrm{AHC}])$ foram comparados (instrumentos-teste). Todos os instrumentos possuem a mesma metodologia de medição 
e utilizam calibradores, controles e insumos do mesmo fabricante.

Seguindo o procedimento adaptado dos documentos EP9-A2 (Method comparison and bias estimation using patient samples) e GP29-A (Assessment of laboratory tests when proficiency testing is not available), propostos pelo Clinical and Laboratory Standards Institute (CLSI) ${ }^{(6,7)}$, foram analisadas 24 amostras de sangue periférico coletadas em tubos contendo ácido etilenodiaminotetracético (EDTA). As amostras foram selecionadas visando obter valores próximos dos diferentes níveis de decisão terapêutica ${ }^{(19)}$. Foram processadas seis amostras por dia, durante quatro dias, em duplicata nos três aparelhos, utilizando o modo fechado, totalizando 48 replicatas em cada instrumento. Foi avaliada a correlação entre os resultados dos parâmetros hematológicos: contagem total de leucócitos (LEU), contagem de hemácias (HEM), hemoglobina (HGB), hematócrito (HTC), volume corpuscular médio (VCM), hemoglobina corpuscular média (HCM), concentração corpuscular média de hemoglobina $(\mathrm{CHCM})$, red cell distribuition width (RDW), contagem de plaquetas (PLT) e volume plaquetário médio (VPM).

A correlação entre os resultados obtidos com os equipamentos-teste (AHB e AHC) e o de referência (AHA) foi avaliada por meio de regressão linear simples (mínimos quadrados) e cálculo do coeficiente de correlação (r). O nível de correlação especificado no presente estudo foi $0,975^{(7)}$. Também foi calculado o coeficiente de determinação $\left(r^{2}\right)$, que mede o percentual da variabilidade do método-teste que é explicada pela variabilidade do método de referência ${ }^{(9)}$. $O r^{2}$ verifica se o modelo de regressão se ajusta satisfatoriamente ao conjunto de dados; um bom ajuste do modelo deve se refletir em um valor de $r^{2}$ próximo de um.
Para que os resultados obtidos nos diferentes equipamentos sejam considerados equivalentes, a diferença entre os resultados (médias das duplicatas) dos instrumentos AHB e AHC (testes) e do instrumento AHA (referência) tem de ser próxima de zero. O gráfico das diferenças permite a inspeção visual dos dados, pois apresenta a diferença entre os resultados do equipamento-teste menos os resultados obtidos com o equipamento-referência (eixo y) versus os resultados do equipamento-referência (eixo $\mathrm{x}$ ). Essas diferenças (bias) devem estar dispersas em torno da linha de diferença zero entre o sistema de teste e o de referência. Para que sejam considerados comutáveis, os resultados do método-teste não devem se desviar dos resultados do método-referência mais do que o definido pelas especificações da qualidade analítica para o erro total. As especificações de imprecisão, bias e erro total utilizadas foram oriundas dos componentes da variação biológica, conforme apresentado na Tabela $1{ }^{(17)}$. A análise foi feita utilizando o programa Excel (Microsoft, 2003, EUA), seguindo as orientações do CLSI(6-8).

\section{Resultados}

As Tabelas $\mathbf{2}$ e $\mathbf{3}$ mostram a correlação dos resultados dos parâmetros hematológicos entre os analisadores AHB e AHC (teste) e o AHA (referência), respectivamente. São apresentados também os erros sistemáticos e totais encontrados e aqueles permitidos neste estudo.

A Figura 1 apresenta os gráficos da diferença entre 0 instrumento-teste $A H B$ e o instrumento-referência (AHA) para LEU, HGB, RDW e PLT, enquanto a Figura 2, os gráficos da diferença entre o instrumento-teste $\mathrm{AHC}$ e o instrumento-referência (AHA).

\section{Tabela 1 Componentes da variação biológica e especificações desejáveis da qualidade analítica ${ }^{(17)}$}

\begin{tabular}{lccccc}
\hline \multicolumn{1}{c}{ Parâmetro } & CVi & CVg & CVa & Bias (\%) & Erro total (\%) \\
Leucócitos & 10,9 & 19,6 & 5,5 & 5,6 & 14,6 \\
Hemácias & 3,2 & 6,1 & 1,6 & 1,7 & 4,4 \\
Hemoglobina & 2,8 & 6,6 & 1,4 & 1,8 & 4,1 \\
Hematócrito & 2,8 & 6,4 & 1,4 & 1,7 & 4,1 \\
VCM & 1,3 & 4,8 & 0,7 & 1,2 & 2,3 \\
HCM & 1,6 & 5,2 & 0,8 & 1,4 & 2,7 \\
CHCM & 1,7 & 2,8 & 0,9 & 0,8 & 2,2 \\
RDW & 3,5 & 5,7 & 1,8 & 1,7 & 4,6 \\
Plaquetas & 9,1 & 21,9 & 4,6 & 5,9 & 13,4 \\
VPM & 4,3 & 8,1 & 2,2 & 2,3 & 5,8 \\
\hline
\end{tabular}

CVi: variação biológica intraindividual; CVg: variação biológica intragrupo; CVa: coeficiente de variação analítica; VCM: volume corpuscular médio; HCM: hemoglobina corpuscular média; CHCM: concentração corpuscular média de hemoglobina; RDW: red cell distribuition width; VPM: volume plaquetário médio. 
Tabela 2 Correlação dos resultados dos parâmetros hematológicos entre AHB (teste) e AHA (referência)

\begin{tabular}{lcccccc}
\hline Parâmetros & $\begin{array}{c}\text { Coeficiente } \\
\text { de correlação } \\
(r)\end{array}$ & $\begin{array}{c}\text { Coeficiente de } \\
\text { determinação } \\
\left(r^{2}\right)\end{array}$ & $\begin{array}{c}\text { Bias médio } \\
\text { encontrado } \\
(\%)\end{array}$ & $\begin{array}{c}\text { Bias } \\
\text { permitido } \\
(\%)\end{array}$ & $\begin{array}{c}\text { Erro total } \\
\text { encontrado } \\
(\%)\end{array}$ & $\begin{array}{c}\text { Erro total } \\
\text { permitido } \\
(\%)\end{array}$ \\
Leucócitos & 0,999 & 0,998 & 3,08 & 5,60 & 6,81 & 14,60 \\
Hemácias & 1 & 1 & 2,32 & 1,70 & 3,85 & 4,40 \\
Hemoglobina & 1 & 1 & 0,90 & 1,80 & 3,53 & 4,10 \\
Hematócrito & 0,999 & 0,998 & 0,74 & 1,70 & 2,45 & 4,10 \\
VCM & 0,990 & 0,980 & 1,58 & 1,20 & 3,22 & 2,30 \\
HCM & 0,994 & 0,988 & 3,44 & 1,40 & 5,57 & 2,70 \\
CHCM & 0,936 & 0,876 & 1,75 & 0,80 & 3,97 & 2,20 \\
RDW & 1 & 1 & 1,09 & 1,70 & 2,53 & 4,60 \\
Plaquetas & 0,999 & 0,998 & 1,06 & 5,90 & 11,89 & 13,40 \\
VPM & 0,984 & 0,968 & 1,75 & 2,30 & 3,09 & 5,80 \\
\hline
\end{tabular}

AHB: analisador hematológico B; AHB: analisador hematológico A; VCM: volume corpuscular médio; HCM: hemoglobina corpuscular média; CHCM: concentração corpuscular média de hemoglobina; RDW: red cell distribuition width; VPM: volume plaquetário médio.

\section{Tabela 3 Correlação dos resultados dos parâmetros hematológicos entre AHC (teste) e AHA (referência)}

\begin{tabular}{lcccccc}
\hline Parâmetros & $\begin{array}{c}\text { Coeficiente de } \\
\text { correlação } \\
(r)\end{array}$ & $\begin{array}{c}\text { Coeficiente de } \\
\text { determinação } \\
\left(r^{2}\right)\end{array}$ & $\begin{array}{c}\text { Bias médio } \\
\text { encontrado } \\
(\%)\end{array}$ & $\begin{array}{c}\text { Bias } \\
\text { permitido } \\
(\%)\end{array}$ & $\begin{array}{c}\text { Erro total } \\
\text { encontrado } \\
(\%)\end{array}$ & $\begin{array}{c}\text { Erro total } \\
\text { permitido } \\
(\%)\end{array}$ \\
Leucócitos & 0,999 & 0,998 & 4,37 & 5,60 & 12,37 & 14,60 \\
Hemácias & 1 & 1 & 2,60 & 1,70 & 4,53 & 4,40 \\
Hemoglobina & 1 & 1 & 1,21 & 1,80 & 3,14 & 4,10 \\
Hematócrito & 1 & 1 & 1,71 & 1,70 & 3,51 & 4,10 \\
VCM & 0,998 & 0,996 & 0,88 & 1,20 & 2,14 & 2,30 \\
HCM & 0,996 & 0,992 & 1,38 & 1,40 & 3,79 & 2,70 \\
CHCM & 0,973 & 0,946 & 0,47 & 0,80 & 2,52 & 2,20 \\
RDW & 0,999 & 0,998 & 0,15 & 1,70 & 0,96 & 4,60 \\
Plaquetas & 0,999 & 0,998 & 3,18 & 5,90 & 10,42 & 13,40 \\
VPM & 0,989 & 0,978 & 2,57 & 2,30 & 5,19 & 5,80 \\
\hline
\end{tabular}

AHC: analisador hematológico C; AHB: analisador hematológico A; VCM: volume corpuscular médio; HCM: hemoglobina corpuscular média; CHCM: concentração corpuscular média de hemoglobina; RDW: red cell distribuition width; VPM: volume plaquetário médio.

\section{Discussão}

Na comutatividade, o objetivo é verificar se os resultados entre os diferentes sistemas analíticos são concordantes ou equivalentes, assim os resultados dos instrumentos-teste não devem se desviar dos resultados do instrumento comparativo mais que os valores definidos pelo controle da qualidade analítica para erro total, erro aleatório (precisão) e erro sistemático (bias ou exatidão). Os erros aleatório, sistemático e total aceitáveis de um sistema analítico podem ser especificados com base na variação biológica, que é definida como a flutuação fisiológica dos constituintes dos fluidos orgânicos em torno de seus pontos homeostáticos ${ }^{(15)}$. Para diversos ensaios, já existem especificações desses parâmetros que permitem avaliar o nível de desempenho de sistemas analíticos ${ }^{(4)}$.

O modelo de utilização dos componentes da variação biológica para definir as especificações da qualidade analítica é compatível com o padrão de desempenho das tecnologias atuais e é recomendado por especialistas dedicados ao estudo da qualidade no laboratório clínico ${ }^{(1,9,10,15,17)}$. Outros critérios podem se basear na relevância clínica ou na variação 

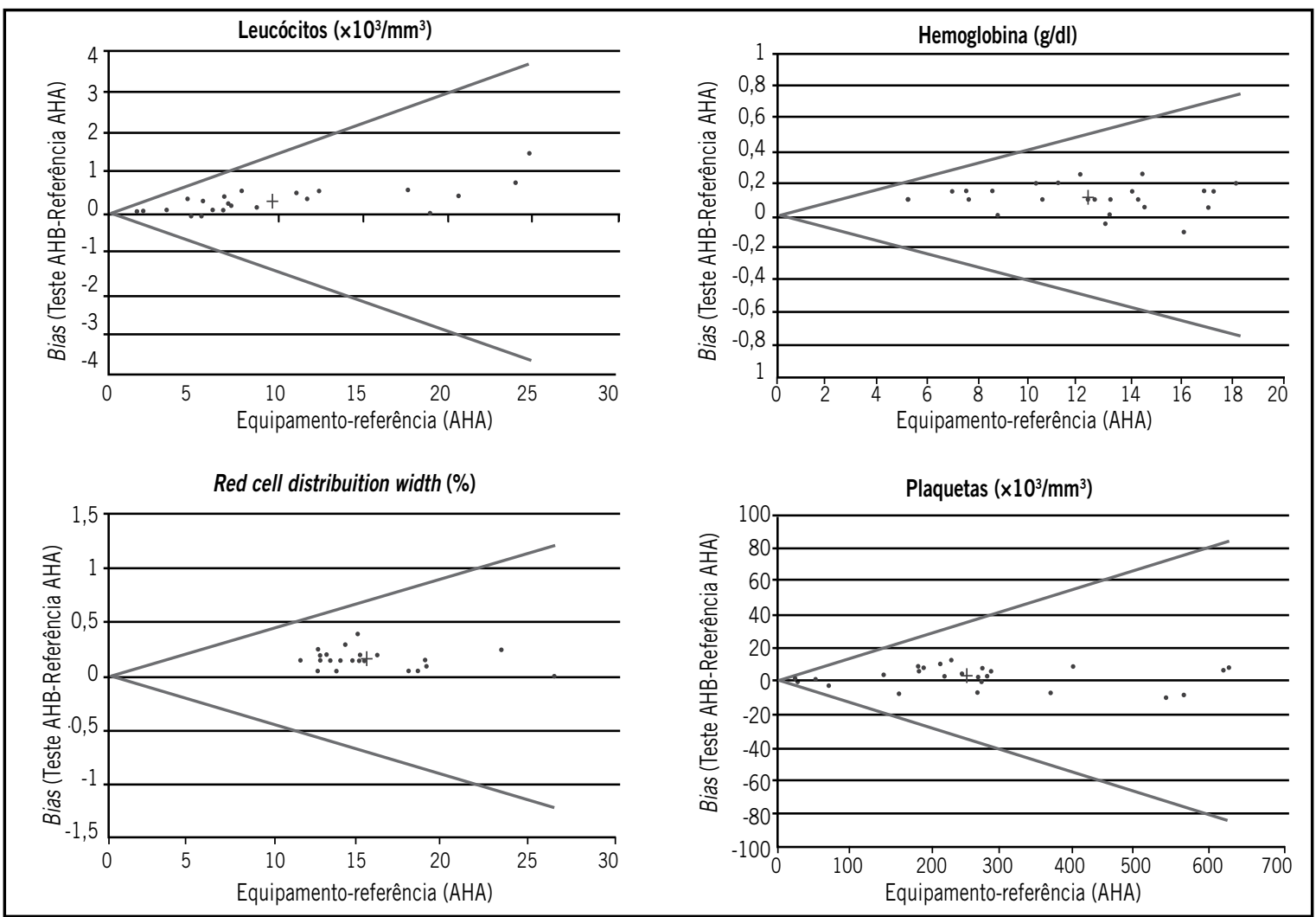

Figura 1 - Diferenças de parâmetros hematológicos entre $A H B$ e $A H A$

A linha < corresponde ao erro total permitido. 0 símbolo + corresponde ao bias médio. $A H B$ : analisador hematológico $B ; A H A$ : analisador hematológico $A$.
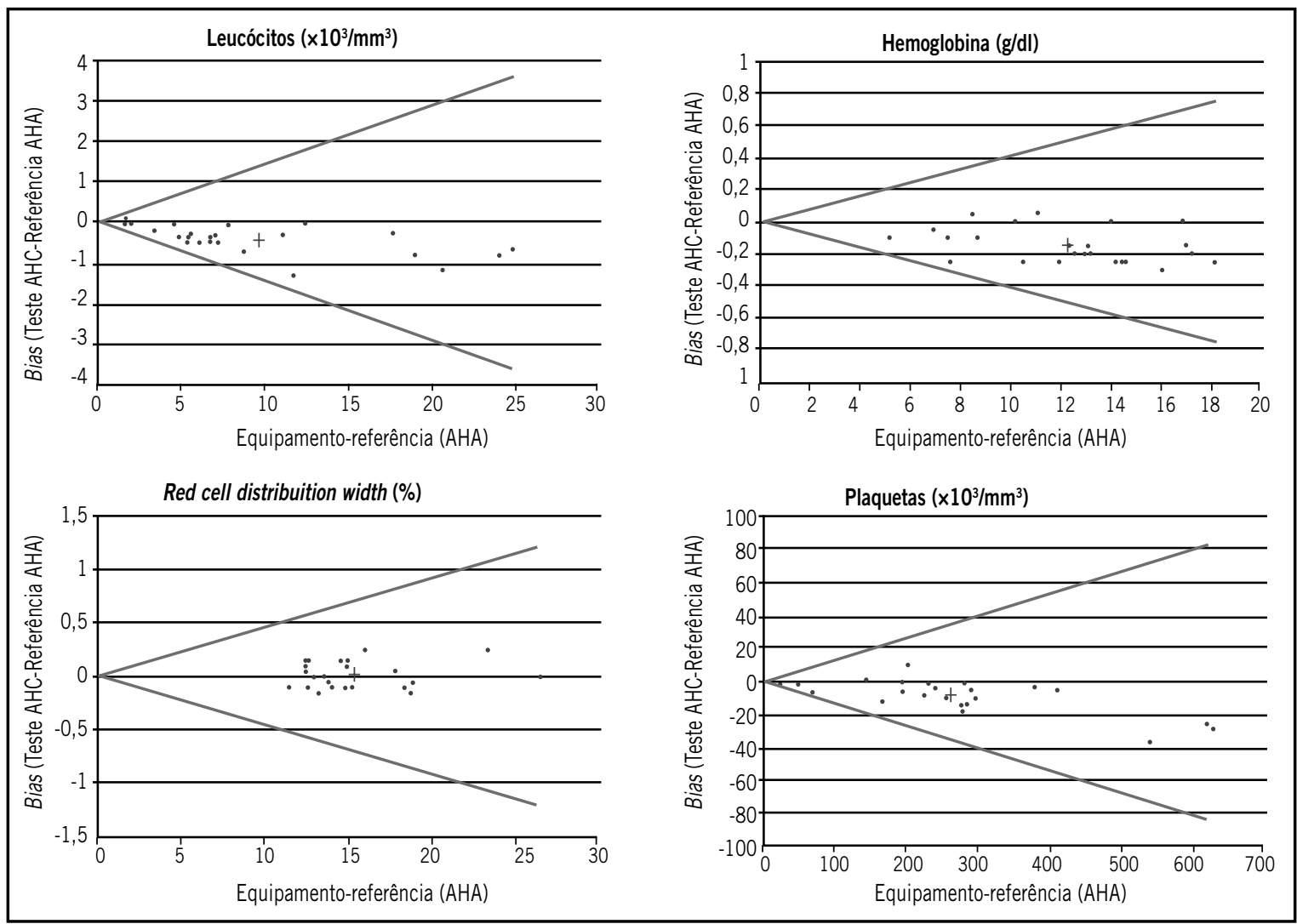

Figura 2 - Diferenças de parâmetros hematológicos entre AHC e AHA

A linha < corresponde ao erro total permitido. 0 símbolo + corresponde ao bias médio.

AHC: analisador hematológico C; AHA: analisador hematológico A. 
mediana dos resultados dos laboratórios ao longo do tempo ${ }^{(9)}$.

As variações analítica e biológica são observadas quando se realiza o mesmo teste em vários indivíduos com intervalos de tempo entre as medições e, aplicados os procedimentos para minimizar os efeitos dos fatores pré-analíticos de variação, observa-se que a concentração dos analitos em cada indivíduo será diferente entre si. Essa diferença de concentração é decorrente das variações analítica e biológica. Se aplicar um procedimento que minimiza o efeito da variação analítica, conhecida pelo controle interno da qualidade, permanecerá ainda uma diferença entre os resultados do mesmo indivíduo. Essa variação residual é denominada variação biológica intraindividual. Quando aplicado o mesmo procedimento em vários indivíduos, observa-se que a variabilidade da concentração dos analitos será diferente entre si. Essa diferença entre indivíduos é denominada variação biológica interindividual ou intragrupo (1).

Os componentes da variação biológica podem ser utilizados para vários propósitos, destacando-se ${ }^{(10)}$ :

- o estabelecimento das especificações da qualidade;

- a avaliação do significado de mudanças na concentração de resultados obtidos seriadamente;

- a avaliação da utilidade dos valores de referência populacionais;

- a escolha do melhor modelo para reportar os resultados;

- a comparação de sistemas analíticos;

- a avaliação da utilidade clínica dos testes de laboratório.

$\mathrm{O}$ instrumento-referência AHA apresentou excelente desempenho de acordo com a análise dos dados dos programas de controle da qualidade do laboratório: Proficiência em Ensaios Laboratoriais (PELM) da SBPC/ML e Programa de Acreditação do College of American Pathologists (CAP). Ao avaliar o equipamento de mesma marca e modelo (Sysmex XE-2100D), Westgard ${ }^{(20)}$ relatou que o mesmo apresenta desempenho de classe internacional, considerando todos os parâmetros, e em todos os níveis de decisão.

A correlação entre os equipamentos avaliados foi ótima para os parâmetros investigados; os valores dos coeficientes de correlação encontrados foram superiores a 0,975 , com exceção da CHCM. Considerando que o erro entre os modelos têm média nula e distribuição normal e pressupondo o método de referência, foi possível usar a regressão linear simples. Os coeficientes de determinação obtidos $\left(r^{2} \cong 1\right)$ validam o modelo de regressão aplicado ao conjunto de dados. Outros dois modelos matemáticos disponíveis para análises de regressão são: regressão de Deming, que utiliza algoritmo de regressão ortogonal, assumindo que a imprecisão está presente em ambos os métodos comparados; regressão de Passing-Bablok, procedimento não paramétrico para regressão linear que não considera a distribuição das amostras nas duas variáveis e a medida de $\operatorname{erros}^{(9)}$.

Os erros sistemático (bias médio) e total obtidos para os parâmetros analisados, quando os equipamentos $\mathrm{AHB}$ e $\mathrm{AHC}$ foram comparados com o AHA, atenderam às especificações da qualidade definidas para o presente estudo. Considerando essas especificações, observamos que o bias médio e o erro total obtido para a maioria dos parâmetros foram inferiores às especificações desejáveis oriundas da variação biológica. Para VCM, HCM e CHCM foram atendidas às especificações mínimas; como esses são parâmetros calculados, dependentes, portanto, da relação com outros parâmetros, especificações menos restritiva podem ser utilizadas.

Assim, exames realizados nos equipamentos descritos neste estudo são comparáveis, podendo ser utilizados indistintamente no acompanhamento de pacientes, em pesquisas clínicas, entre outras situações, minimizando a interferência da variabilidade analítica nos resultados.

\section{Conclusão}

Laboratórios que realizam os mesmos exames com diferentes métodos, sistemas ou equipamentos devem realizar a verificação da comutatividade. Assim, as ações de controle da qualidade dos laboratórios clínicos devem contemplar a realização dessa avaliação, sua periodicidade e as especificações da qualidade analítica. O presente estudo, utilizando procedimento padronizado internacionalmente, prático e de baixa complexidade, avaliou a comutatividade ou equivalência dos resultados de exames hematológicos realizados em equipamentos em um laboratório público de um hospital universitário. Foi demonstrada a factibilidade de realização da avaliação da comutatividade entre as ações da gestão da qualidade em laboratórios clínicos.

\section{Agradecimentos}

Agradecemos à Coordenação de Aperfeiçoamento de Pessoal de Nível Superior (CAPES), pela bolsa de mestrado da aluna Chams B. Maluf, à Fundação de Amparo à Pesquisa de Minas Gerais (FAPEMIG), pela bolsa de iniciação científica de Indiayara O. Silva, e ao Setor de Hematologia do Serviço de Medicina Laboratorial do Hospital das Clínicas da Universidade Federal de Minas Gerais. 


\section{Referências}

1. BASQUES, J. C. Especificações da qualidade analítica. Belo Horizonte: Labtest, 2009. Disponível em: <http:// www.labtest.com.br/publicacoes/publicacoeslabtest>. Acesso em: 12 dez. 2010.

2. INSTITUTO BRASILEIRO DE GEOGRAFIA E ESTATÍSTICA (IBGE). Estatísticas da saúde: assistência médicosanitária 2009. Rio de janeiro: IBGE, 2010. Disponível em: <http://www.ibge.gov.br/home/estatistica/ populacao/condicaodevida/ams/2009/ams2009.pdf>. Acesso em: 9 ago. 2011.

3. INTERNATIONAL ORGANIZATION OF STANDARDIZATION (ISO). International vocabulary of basic and general terms in metrology. Geneve, Switzerland. 1993.

4. KLEE, G. A conceptual model for establishing tolerance limits for analytic bias and imprecision based on variations in population test distributions. Clin Chim Acta, v. 260, n. 2, p. 175-88, 1997.

5. MILLER, W. G. The role of proficiency testing in achieving standardization and harmonization between laboratories. Clin Biochem, v. 42, n. 4-5, p. 232-5, 2009.

6. NCCLS/CLSI. Assessment of laboratory tests when proficiency testing is not available. Approved guideline. Document G29-A. 2002.

7. NCCLS/CLSI. Method comparison and bias estimation using patient samples. Approved guideline. Document EP9-A2. 2002.

8. NCCLS/CLSI. User demonstration of performance for precision and accuracy. Approved guideline. Document EP15-A. 2001.

9. OLIVEIRA, C. A.; MENDES, M. E. (Org.). Gestão da fase analítica do laboratório: como assegurar a qualidade na prática. 1. ed. Rio de Janeiro: ControlLab, 2010.

10. PETERSEN, P. H.; FRASER, C. G. Strategies to set global analytical quality specifications in laboratory medicine: 10 years on from the Stockholm consensus conference. Accred Qual Assur, v. 15, n. 6, p. 323-30, 2010.
11. PROGRAMA DE ACREDITAÇÃO PARA LABORATÓRIOS CLÍNICOS (PALC) 2007. Disponível em: <http://www. sbpc.org.br/upload/conteudo/320070815172544. pdf >. Acesso em: 12 dez. 2010.

12. PROGRAMA DE ACREDITAÇÃO PARA LABORATÓRIOS CLÍNICOS (PALC) 2010. Disponível em: <http://www. sbpc.org.br/upload/conteudo/320101013112151. pdf>. Acesso em: 12 dez. 2010.

13. PROGRAMA DE ACREDITAÇÃO PARA LABORATÓRIOS CLÍNICOS (PALC) 2010. Disponível em: <http://www. sbpc.org.br/laboratorio.php>. Acesso em: 10 jan. 2011.

14. REJ, R. Accurate enzyme-activity measurement: 2 decades of development in the commutability of enzyme qualitycontrol materials. Arch Pathol Lab Med, v. 117, n. 4, p. 352-64, 1993

15. RICOS, C. et al. Procedure for studying commutability validated by biological variation. Clin Chim Acta, v. 268, n. 1-2, p. 73-83, 1997.

16. SCIACOVELLI, L. et al. The role of the external quality assessment. Biochem Med, v. 20, n. 2, p. 160-4, 2010.

17. SOCIEDAD ESPAÑOLA DE BIOQUÍMICA CLÍNICA Y PATOLOGIA MOLECULAR. Base de datos de Variación biológica. Disponível em: <http://www. seqc.es/es/Sociedad/7/51/102/Base_de_datos_de_ Variacion_biologica_Bases_de_datos_y_documentos_ del_Comite_de_Garantia_de_la_Calidad_y_ Acreditacion_Comite_de_Garantia_de_la_Calidad_y_ Acreditacion_/>. Acesso em: 10 fev. 2010.

18. WESTGARD, J. O. Internal quality control: planning and implementation strategies. Ann Clin Biochem, v. 40, p. 593-611, 2003.

19. WESTGARD, J. O. Medical decision levels. 2009. Disponível em: <http://www.westgard.com/decision. htm/\#hematology>. Acesso em: 10 fev. 2010.

20. WESTGARD, J. O. Sysmex XE 2100. 2007. Disponível em: $<\mathrm{http}$ ://www.westgard.com/qcapp41.htm>. Acesso em: 10 jan. 2011.

\begin{tabular}{l|l} 
& Endereço para correspondência \\
\hline & Pedro Guatimosim Vidigal \\
Faculdade de Medicina da UFMG \\
Av. Prof. Alfredo Balena, 190, sala 403 - Santa Efigênia \\
CEP: 30130-100 - Belo Horizonte-MG \\
Tel: (31) 3409-9774 \\
Fax: (31) 3409-9782 \\
e-mail: pedrovidiga@@ufmg.br
\end{tabular}

